\begin{tabular}{|c|c|}
\hline Title & Scale calcification in the goldfish in vitro : histological and quantitative analysis \\
\hline Author(s) & Ogawa, Nobuhiro; Ura, Kazuhiro; Takagi, Y asuaki \\
\hline Citation & $\begin{array}{l}\text { Fisheries Science, } 76(2), 189-198 \\
\text { https://doi.org/10.1007/\$12562-009-0197-7 }\end{array}$ \\
\hline Issue Date & $2010-03$ \\
\hline Doc URL & http:/hdl.handle.net/2115/43065 \\
\hline Rights & $\begin{array}{l}\text { ○ } 2010 \text { 公益社団法人日本水産学会; ○ } 2010 \text { The Japanese Society of Fisheries Science; The final publication is } \\
\text { avail able www.springerlink.com }\end{array}$ \\
\hline Type & article (author version) \\
\hline File Information & FS76-2_189-198.pdf \\
\hline
\end{tabular}

Instructions for use 


\section{Scale calcification in the goldfish in vitro: histological and quantitative analysis}

Nobuhiro Ogawa* · Kazuhiro Ura · Yasuaki Takagi

Graduate School of Fisheries Sciences, Hokkaido University, 3-1-1 Minato-cho, Hakodate, Hokkaido 041-8611, Japan

\footnotetext{
*Corresponding Author; $\quad$ Nobuhiro Ogawa

Graduate School of Fisheries Sciences

Hokkaido University

3-1-1 Minato-cho, Hakodate

Hokkaido 041-8611

Japan

TEL/FAX: +81-138-40-5551

e-mail: n-ogawa@fish.hokudai.ac.jp
} 
Abstract The external layer of a teleost fish scale is composed of type I collagen, an amorphous matrix substance and hydroxyapatite crystals. Calcification of this layer can be inhibited in the scale regenerating process under a calcium and phosphate deficient (CaDPD) condition, and can be facilitated by incubation in physiological saline. The aim of this study was to evaluate this model of calcification using histological and quantitative analysis in order to promote further understanding of the mechanism of calcification in fish scales. We found that the external layer of the scales produced under a CaDPD condition contained more densely-aligned collagen fibrils with a small amount of the amorphous matrix substance. The CaDPD scale contained only one-third of the amount of calcium and phosphate present in the control fish. After 4-hour incubation, a 2-3-fold increase in calcium content and a 1.5-fold increase in phosphate content were observed. Calcification proceeded in the external layer, and mineral deposits grew only in the electron-dense matrix substance. We conclude that this model would be suitable to study the early process of fish scale calcification that occurs in the non-collagenous substance. The electron-dense substance may contain key molecules that promote calcification.

194/ 200 word

Keywords biomineralization · Carasssius auratus · goldfish · in vitro calcification · scale regeneration 


\section{Introduction}

The inner most layer of the teleost fish scale, the basal plate, is a collagenous plate having a unique fibril-alignment similar to mammalian cornea. In both basal plate and cornea, multiple collagen layers are stacked, and the fibrils in one layer are parallel, but those in different layers are orthogonal [1-4]. As a result, the layers form a plywood-like structure. Because of extremely high regeneration ability of the scale, the process of scale regeneration is a good model with which to study the cellular mechanism to construct cornea-like collagenous extracellular matrix [5]. Clarification of the mechanism is a prerequisite for bioinspired fabrication of artificial collagenous matrices to substitute injured cornea for the regenerative medicine [5]. Our research is now concerned with the fabrication of such matrices using scale collagen since it has low risk of zoonotic infection compared with the current major collagen source (bovine and porcine) for medical use.

Another layer of the scale, the external layer, has a structure similar to that of fibrous bone or dentine. It consists of calcium phosphate hydroxyapatite crystals, collagen (mainly type I), and amorphous non-collagenous substances [6-9]. Therefore, the process of regeneration of the external-layer would be a good model with which to study the cellular mechanism to construct a bone/dentine-like, calcified collagenous matrix. Bone is a tissue that has a limited ability of regeneration, and thus, bone substitutes are often required for clinical treatment. Although fabrication of artificial materials suitable for such purpose has long been attempted, at present there is no satisfactory one that has an excellent biocompatibility, high osteoconductivity and bioactivity. In order to fabricate high-performance bone substitutes, it is important to mimic the composition and organization of bone extracellular matrix, which is generally an important determinant of cellular behavior such as cellular adhesion, migration, proliferation and differentiation [10]. Regulation of size and organization of hydroxyapatite crystals is quite important, as can be inferred from the fact that gene expression patterns related to osteoblast adhesion, proliferation, extracellular matrix synthesis and diferentiation were regulated in part by the size of sieved hydroxyapatite in MC3T3-E1 mouse osteoblastic cells [11]. However, studies on the mechanism of scale regeneration including early mineral deposition are scarce, except for histological observations of the regenerating process. For this reason, the physiological, biochemical or molecular understanding of this process remains incomplete.

Iguchi [12] and Yamane et al. [13] reported that fish reared in the distilled water and fed a calcium and phosphate deficient diet (CaDPD condition) regenerate uncalcified scales, and that these uncalcified regenerating scales can subsequently be artificially calcified in vitro. This technique is useful to investigate the precise 
physiological and molecular mechanisms underlying early mineral deposition. However, the structure of the scales regenerated under the CaDPD condition, the calcification levels of the uncalcified and artificially calcified scales, and the ultrastructure of mineral deposits and their surrounding areas after incubation are unknown. For these reasons, this model cannot yet be accurately applied to studies of calcification mechanisms.

In this study, we have re-evaluated this mineralization model. First, we quantified the calcium and phosphate levels of scales regenerated under the CaDPD condition. Second, we evaluated the effects of incubation in physiological saline on the calcium and phosphate levels of the regenerating scales. Thereafter, light and electron microscopic observations were conducted to compare the structure and mineralization state of scales regenerated under control and CaDPD conditions, and those of regenerated scales that were subsequently calcified in vitro.

\section{Materials and methods}

Fish

Goldfish Carassius auratus (weighing about 48.4 g) were purchased from a commercial dealer. They were kept in tap water at $26^{\circ} \mathrm{C}$ and fed a commercial diet (4C; Nippon Formula Feed Mgf Co., Ltd., Kanagawa, Japan) 4 times a day (total $4 \%$ of body weight/day) for at least 1 week before the experiment.

Experimental diet

A calcium- and phosphate-containing diet (control diet), and a calcium- and phosphate-deficient diet (CaDPD diet) were prepared largely by following the method of Takagi et al. [14]. The composition of the two diets are listed in Table 1. The calcium content of the control and the CaDPD diet was 15.64 and $0.49 \mathrm{mg} / \mathrm{g}$ dry weight, respectively. The phosphate content of the control and the CaDPD diet were 42.3 and $1.78 \mathrm{mg} / \mathrm{g}$ dry weight, respectively.

Experimental protocol

After the fish were anesthetized in a $0.02 \%$ solution of 2-phenoxyethanol, every second scale was removed from 
the both entire flanks. Next, fish were divided into 2 groups, each containing 8 fish. Fish in the first group ('control fish') were reared in tap water at $26^{\circ} \mathrm{C}$ and fed the control diet 4 times a day (a total of $4 \%$ of body weight/day). Fish in the second group ('CaDPD fish') were reared in deionized water at $26^{\circ} \mathrm{C}$ and fed the CaDPD diet 4 times a day (a total of $4 \%$ of body weight/day). After 7 days, the fish were anesthetized and all of the regenerating scales were collected; these scales were called the ' 1 st-trial scales'. The fish were then reared for another 7 days in each condition, and all regenerating scales were collected again ('2nd-trial scales'). The fish were reared for a further 7 days in each condition, and the regenerating scales were obtained ('3rd-trial scales'). The regenerating scales collected were subjected to calcium and phosphate content analysis, and light and electron microscopy. A portion of the regenerating scales was also used for the in vitro calcification experiment.

In vitro calcification

A portion of the regenerating scales was incubated in physiological saline $\left(\mathrm{Na}^{+}: 154 \mathrm{mM} ; \mathrm{K}^{+}: 7 \mathrm{mM} ; \mathrm{Ca}^{2+}: 2.5\right.$ $\mathrm{mM} ; \mathrm{Mg}^{2+}: 1.5 \mathrm{mM} ; \mathrm{PO}_{4}{ }^{3-}: 2 \mathrm{mM}$; glucose: $10 \mathrm{mM}$; HEPES: $10 \mathrm{mM}$; $\mathrm{pH} 7.4$ ) for 4 hours at $37^{\circ} \mathrm{C}$. After this incubation, the scales were subjected to calcium and phosphate content analysis, and light and electron microscopy.

Ca and Pi content analysis

The scales were dried overnight at $80^{\circ} \mathrm{C}$, weighed and solubilized in concentrated $\mathrm{HNO}_{3}$ at $40^{\circ} \mathrm{C}$. Calcium levels were measured by a Hitachi Z-6100 polarized Zeeman atomic absorption spectrophotometer (Tokyo, Japan). Inorganic phosphate levels were determined by following the method of Goldenberg and Fernandez [15], using a Hitachi U-2000 spectrophotometer (Tokyo, Japan).

Light microscope observation

The regenerating scales were fixed in $70 \%$ ethanol and divided into two groups. The scales in one group were immersed in a $1 \%$ silver nitrate solution and illuminated with fluorescent light for 10 minutes to detect calcification site. Next, they were rinsed in deionized water, mounted on glass slides and examined with a Nikon 
eclipse E800 light microscope (Tokyo, Japan). The scales in the other group were dehydrated in a graded series of ethanol and embedded in JB-4 resin (Polysciences, Inc.). Frontal sections (3 $\mu$ m thickness) were cut with a Reichert-Jung 2050 microtome (Heidelberg, Germany), stained with 1\% silver nitrate and a mixture of methylene blue and azure II, and observed under a light microscope.

Transmission electron microscope (TEM) observation

The scales were immersed in a mixture of $2 \%$ paraformaldehyde and $2.5 \%$ glutaraldehyde in $0.05 \mathrm{M}$ cacodylate buffer ( $\mathrm{pH}$ 7.6) for $2 \mathrm{~h}$ at room temperature. After a short rinse in buffer, the samples were postfixed for $1 \mathrm{~h}$ in $1 \% \mathrm{OsO}_{4}$ in $0.05 \mathrm{M}$ cacodylate buffer ( $\mathrm{pH}$ 7.6). The samples were then rinsed in buffer, dehydrated in a graded series of ethanol, and embedded in Spurr resin (Agar scientific Ltd, Cambridge Road, UK). Ultrathin sections were cut with a Reichert-Nissei Ultracut N ultramicrotome (Heidelberg, Germany) using a diamond knife. Sections were unstained or stained with uranyl acetate and lead citrate, and examined with a Hitachi H-7000 transmission electron microscope (Tokyo, Japan).

\section{Statistics}

Student's $t$-test for unpaired data was used to test the significance of the means between the control and CaDPD group. Student's t-test for paired data was used to test the significance of the means of the calcium and phosphate content of the scales before and after incubation in the in vitro calcification experiments. Statistical significance was set at $P<0.05$. All data are reported as mean \pm standard error. All analyses were performed with Stat View 5.0 for Windows (SAS Institute Inc.).

\section{Results}

Calcium and phosphate content of the regenerating scales

As shown in Fig. 1, the dry weight of the regenerating scales collected from CaDPD fish was about $80 \%$ of that from control fish in all trials. Figure 2 shows the Ca and Pi content of the regenerating scales before and after 
incubation in the in vitro calcification experiments. Before the incubation, the calcium and phosphate content of the 1st-trial scales from CaDPD fish was about 30\% and 33\%, respectively, of that of 1st-trial scales from control fish. Moreover, the calcium and phosphate content of the 2nd- and 3rd-trial scales from CaDPD fish was even smaller than that of the 1st-trial scales. After the incubation, the calcium and phosphate content of the regenerating scales collected from control fish increased only slightly: the calcium content was significantly higher after the incubation only in the 3rd-trial scales. The calcium content of the scales from CaDPD fish was increased two- to three-fold after the incubation. The phosphate content of the regenerating scales from CaDPD fish was increased about 1.5-fold after the incubation. However, the calcium and phosphate content of the scales from CaDPD fish did not reach the levels in control fish after the incubation.

Light microscopy

Figure 3 shows a surface view of the 1st-trial scales stained with silver nitrate, before and after the incubation. Before the incubation, the control scales showed strong staining with silver nitrate except for the marginal narrow area and grooves. In the scales from CaDPD fish, by contrast, silver nitrate staining was relatively weaker than that in the scales from control fish. In particular, the marginal wide area did not react with silver nitrate. The results were almost the same for the 2nd- and 3rd-trial scales. After the incubation, both control and CaDPD scales reacted well with silver nitrate.

In the histological sections (Fig. 4), the regenerating scales from control fish comprised an outer flat-cell layer, an external layer that reacted with silver nitrate, the basal plate, and an innermost cubic-cell layer. The structure was the same in the scales collected from CaDPD fish, however, the external layer of the regenerating scales from CaDPD fish was scarcely stained with silver nitrate and had more pores and concavities containing several cells. Around the pores and concavities, the silver nitrate staining was even weaker than in the other parts of the external layer. After the incubation, silver nitrate reacted only with the external layer of scales collected from both control and CaDPD fish. The extent of the reaction with the scales of CaDPD fish increased after the incubation.

Transmission electron microscopy

In the regenerating scales collected from control fish, the external layer was morphologically divided into five 
layers, mainly depending on the diameter of the collagen fibrils (Fig. 5). The outermost layer, to which flat cells were attached, was composed of scattered collagen fibrils (about $10 \mathrm{~nm}$ diameter) with an electron-light substance (A layer). The next layer comprised relatively assembled but scattered collagen fibrils with a diameter of about $20 \mathrm{~nm}$ (B layer). The spaces between the fibrils were filled with electron-dense and electron-light amorphous substances. Next to the B layer, a layer with thick collagen fibrils (about $50 \mathrm{~nm}$ diameter) was observed (C layer). The collagen fibrils were aggregated and formed thick bundles. Electron-dense and electron-light substances filled the spaces between the collagen fibrils. This layer was in part patchily distributed in the innermost of the external layer. Under the C layer, the D layer, whose structural features were similar to B layer, was observed. The innermost layer was composed of collagen fibrils (about $50 \mathrm{~nm}$ diameter) that were most aggregated and relatively aligned (E layer). In the CaDPD scales, the amount of non-collagenous matrix in the external layer was much less than that in the control scales, resulting in densely-packed collagen fibrils in the external-layer matrix. Moreover, only the B- and E-like layers were observed. The B-like layer was composed of two parts. One had small amount of electron-dense substances between the fibrils, whereas another had electron-light substances. None of the boundaries between the layers was clear.

To investigate the mineral deposits in the external layer, unstained sections were examined (Fig. 6). In the unstained sections of the control scales, the external layer comprised amorphous electron-dense and electron-light substances, and electron-dense spindle-shaped particles were observed in the electron-dense substance. Both the electron-dense substance and particles were rich in the central region of the external layer where B and C layers might be distributed as observed in the stained sections. In the external layer of the CaDPD scales, by contrast, the electron-dense particles and substances were scarcely observed. After incubation of the CaDPD scales, the electron-dense substance was observed more frequently in the central region of the external layer, where B layer was distributed in the stained sections, and the spindle-shaped particles appeared within this substance. However, the particles were smaller than those in the control scales. The amount of particles in the CaDPD scales was also less than in the control scales.

\section{Discussion}

We have shown here, by both histological and quantitative methods, that fish reared in the CaDPD condition regenerated scales in which calcification was inhibited. It was also observed that calcification of these scales could proceed in physiological saline only in their external layer, as observed in the control regenerating scales 
in vivo. Moreover, electron-dense, spindle-shaped particles, which morphologically resembled mineral deposits present in fish scales [2, 7, 16-18], grew in an electron-dense substance both in the regenerated scales from control fish and in the in vitro incubated scales from CaDPD fish, although the amount of the substance was smaller in the latter. These findings suggest that the mechanisms of calcification induced in the CaDPD scales in vitro and control scales in vivo are comparable, and thus, the CaDPD scale is a good model with which to study the mechanism underlying the early calcification of teleost scales.

The CaDPD scale is composed of an external layer and a basal plate, as is a control regenerating scale. In the CaDPD scale, however, the dry weight was lower and the density of collagen fibrils was higher than that in the control scale. These results indicate that deposition of the scale matrix components, especially that of non-collagenous substances, is inhibited in the CaDPD condition. In addition, Iguchi [12] and Yamane et al. [13] reported that goldfish reared in the CaDPD condition resulted in hypophosphatemia but not hypocalcemia. Under a calcium deficient condition, tilapia Oreochromis niloticus induces calcium mobilization from its scales and bone, and prevent hypocalcemia $[14,19]$. Thus, inhibition of calcification in the CaDPD scales would be caused by both a smaller amount of non-collagenous matrices and hypophosphatamia, but not by hypocalcemia.

Matrix vesicles are the membrane-bound particles located in the extracellular matrix of various calcified tissues. They contain a putative calcification-inducing enzyme, alkaline phosphatase, and are generally accepted as calcification-inducing structures in various calcified tissues in mammals [20-23]. In previous studies of regenerating scales, some researchers have observed matrix-vesicle-like structures with hydroxyapatite-like crystals in the scale marginal area, where calcification initiates [2, 7, 16-18]. However, a mineral deposit in the external layer independent of such structures has also been observed in scales. Olson and Watabe [24] showed that the mineral deposit of developing scales was not related to observable substances or structures in the sheepshead minnow Cyprinodon variegatus. In the developing scales of chum salmon Oncorhynchus keta, goldfish, guppy Lebistes reticulatus and Poecilia reticulata, tilapia tilapia rendalli and rainbow trout Salmo gairdnerii irideus, the mineral deposit is observed in the interfibrilar electron-dense substance [2, 6, 8, 16, 25]. Thus, the matrix vesicles were not prerequisites in scale calcification, as suggested by Bereiter-Hahn and Zylberberg [2]. In this study, by way of the in vitro system, we succeeded to show that the very beginning of calcification in goldfish regenerating scales proceeds in the electron-dense substance independent of matrix vesicles. Therefore, the present in vitro system is suitable to study the mechanism of matrix-vesicle-independent calcification.

Using histochemical methods, Maekawa and Yamada [6] suggested that electron-dense particles, containing 
sulphated mucopolysaccharides and protein-mucopolysaccharide complexes, have a role in nucleation of the mineral phase in the scales of juvenile rainbow trout Salmo gairdnerii irideus. The electron-dense substances that we observed in this study may also be calcification-inducing proteoglycan composites, as suggested by Maekawa and Yamada [6]. It is difficult to imagine that a 4-hour incubation could induce the rapid synthesis of calcification-related organic matrices by scale-forming cells. We therefore propose that these molecules may already exist in the CaDPD scale matrix, dispersed in the interfibrillar spaces, although their amount may be less than in normal scales. Incubation in physiological saline may induce these molecules to aggregate and show electron density, and the process of calcification may be induced by the aggregated macromolecules. The importance of aggregated macromolecules in the initiation of calcification was also reported in mammalian bone. Recently, it has been reported that local extracellular nucleation complexes, called 'biomineralization foci' which are independent structures with matrix vesicles, are composed mainly of bone acidic glycoprotein-75 (BAG-75) and bone sialoprotein in UMR106-01 osteoblastic cultures [26]. BAG-75 has the ability to self-associate into 10-20 $\mu \mathrm{m}$ supramolecular supherical complexes composed of microfibrils [27, 28]. Immunohistological analysis using a BAG-75 aggregation-specific monoclonal antibody showed that a BAG-75 complex exists in vivo at the site of new primary bone formation [28], suggesting that matrix-vesicle-independent calcification occurs also in the mammalian bone. We assume that the electron-dense matrix substance observed in this study may comprise aggregated macromolecules similar to the biomineralization foci in the mammalian bone, and may have important roles in matrix-vesicle-independent calcification of scales.

Calcification-related organic matrices that can promote hydroxyapatite nucleation have long been studied in mammalian calcified tissues. Nevertheless, only a few proteins have been identified as a crystal nucleater [29]. The method developed in this study will be a key tool to identify organic matrices that promote scale calcification, especially in the early stages of calcification. Further biochemical and molecular biological investigations will reveal the molecular composition of the electron-dense substance and the mechanism underlying matrix calcification in detail. Such information will be applicable to the development of artificial bone using scale collagen.

\section{Acknowledgments}

This study was supported in part by Grant-in-Aid for Exploratory Research (No. 16658077) and for $21^{\text {st }}$ century COE program awarded by the ministry of education, Culture, Sports, Science and Technology of Japan. The study was also supported in part by Grant-in-Aid for a Creative Scientific Research (No.17GS0311) and a 
Research Grant for Young Scientists (No. 18-04520) awarded by the Japan Society for Promotion of Science. 


\section{References}

1. Zylberberg L, Géraudie J, Meunier FJ, Sire J-Y (1992) Biomineralization in the integumental skeleton of the living lower vertebrates. In: Hall BK (ed) Bone 4, Bone Metabolism and mineralization. CRC Press, Boca Raton, pp 171-224

2. Bereiter-Hahn J, Zylberberg L (1993) Regeneration of teleost fish scale. Comp Biochem Physiol 105A:625-641

3. Huysseune A, Sire J-Y (1998) Evolution of patterns and processes in teeth and tooth-related tissues in non-mammalian vertebrates. Eur J Oral Sci 106:437-481

4. Sire J-Y, Akimenko M-A (2004) Scale development in fish: a review, with description of sonic hedgehoc (shh) expression in the zebrafish (Danio rerio). Int J Dev Biol 48:223-247

5. Takagi Y, Ura K (2007) Teleost fish scales: a unique biological model for the fabrication of materials for corneal stroma regeneration. J Nanosci Nanotechnol 7:757-762

6. Maekawa K, Yamada J (1970) Some histochemical and fine structural aspects of growing scales of the rainbow trout. Bull Fac Fish Hokkaido Univ 21:70-77

7. Sire J-Y, Géraudie J (1984) Fine structure of regenerating scales and their associated cells in the cichlid Hemichromis bimaculatus (Gill). Cell Tissue Res 237:537-547

8. Zylberberg L, Nicolas G (1982) Ultrastructure of scales in teleosts (Carassius auratus L.) after use of rapid freeze-fixation and freeze-substitution. Cell Tissue Res 223:349-367

9. Sauk JJ, Cocking-Johnson D, Cervenka VA, Van Kampen CL (1984) Noncollagenous phosphoprotein derived from teleostean fish-scales. Biochimi Biophys Acta 798:199-203

10. Ekblom P, Vestweber D, Kemler R (1986) Cell-matrix interactions and cell adhesion during development. Ann Rev Cell Biol 2:27-47

11. Xie J, Baumann MJ, McCabe LR (2004) Osteoblasts respond to hydroxyapatite surfaces with immediate changes in gene expression. J Biomed Mater Res 71A:108-117

12. Iguchi M (1985) Gyoruikousoshiki (hone · uroko) no seichou to sekkaika ni okeru karusiumu to rin no kyodou ni kansuru kenkyu. (Studies on the actions of calcium and phosphate in the growth and calcification of fish bones and scales). PhD Dissertation, Hokkaido University, Hakodate (in Japanese)

13. Yamane S, Iguchi M, Ogasawara T, Nakamura Y (1982) Effects of blockage of exogenous calcium and phosphorus on the calcium regulatory systems in goldfish. Comp Biochem Physiol 72A:709-713

14. Takagi Y, Hirano T, Yamada J (1989) Scale regeneration of tilapia (Oreochromis niloticus) under various 
ambient and dietary calcium concentrations. Comp Biochem Physiol 92A:605-608

15. Goldenberg H, Fernandez A (1966) Simplified method for the estimation of inorganic phosphorus in body fluids. Clin Chem 12: 871-882

16. Yamada J (1971) A fine structural aspect of the development of scales in the chum salmon fry. Nippon Suisan Gakkaishi 37:18-29

17. Fouda MM (1979) Studies on scale regeneration in the Common goby, Pomatoschistus microps (Pisces). J Zool Lond 189:503-509

18. Sire J-Y, Géraudie J (1983) Fine structure of developing scale in the Chichlid Hemichromis bimaculatus (Pisces, Teleostei, Perciformes). Acta Zool 64:1-8

19. Takagi Y, Yamada J (1992) Effect of calcium deprivation on the methabolism of acellular bone in tilapia, Oreochromis niloticus. Comp Biochem Physiol 102A:481-485

20. Anderson HC (1984) Mineralization by matrix vesicles. Scan Electron Micros 2:953-964

21. Anderson HC (1995) Molecular biology of matrix vesicles. Clin Orthop Relat Res 314:266-280

22. Anderson HC (2003) Matrix vesicles and calcification. Curr Rheumatol Rep 5:222-226

23. Arsenault AL, Frankland BW, Ottensmeyer FP (1991) Vectorial sequence of mineralization in the turkey leg tendon determined by electron microscopic imaging. Calcif Tissue Int 48:46-55

24. Olson OP, Watabe N (1980) Studies on formation and resorption of fish scales. IV. Ultrastructure of developing scales in newly hatched fry of the Sheepshead minnow (Cyprinodon variegatus). Cell Tissue Res 211:303-316

25. Schörnbörner AA, Boivin G, Baud CA (1979) The mineralization processes in teleost fish scales. Cell Tissue Res 202:203-212

26. Huffman NT, Keightley JA, Chaoying C, Midura RJ, Lovitch D, Veno PA, Dallas SL, Gorski JP (2007) Association of specific proteolytic processing of bone sialoprotein and bone acidic glycoprotein-75 with mineralization within biomineralization foci. J Biol Chem 282:26002-26013

27. Gorski JP, Kremer EA, Chen Y (1996) Bone acidic glycoprotein-75 self-associates to form large macromolecular complexes. Connect Tissue Res 35:137-143

28. Gorski JP, Kremer EA, Chen Y, Ryan S, Fullenkamp C, Delviscio J, Jensen K, McKee MD (1997) Bone acidic glycoprotein-75 self-associates to form macromolecular complexes in vitro and in vivo with the potential to sequester phosphate ions. J Cell Biochem 64:547-564

29. Hunter GK, Hauschka PV, Poole AR, Rosenberg LC, Goldenberg HA (1996) Nucleation and inhibition of 
hydroxylapatite formation by mineralized tissue proteins. Biochem J 317:59-64

30. Halver JE, Coastes JA (1957) A vitamin test diet for long-term feeding studies. Prog Fish Cult 19:112-118 


\section{Figure captions}

Fig. 1 Effect of the calcium and phosphate deficient (CaDPD) condition on the dry weight of 7-day regenerating scales. Values are means \pm SE for 7 samples. *Significantly different $(P<0.05)$ by unpaired $t$-test

Fig. 2 Calcium (a-c) and phosphate (d-f) content of 7-day regenerating scales before and after incubation in physiological saline for 4 hours at $37^{\circ} \mathrm{C}$. Regenerating scales were collected from calcium and phosphate sufficient (control) or deficient (CaDPD) conditions. Values are means \pm SE for 7 samples. *Significantly different $(P<0.05)$ by paired $t$-test. $\quad$ †Significantly different $(P<0.05)$ by unpaired $t$-test

Fig. 3 Light microscopy images of the surface view of 7-day regenerating scales. a A scale regenerated in the control condition, $\mathbf{b}$ a scale regenerated in the control condition and incubated for 4 hours in physiological saline, c a scale regenerated in the CaDPD condition, $\mathbf{d}$ a scale regenerated in the CaDPD condition and incubated for 4 hours in physiological saline. Arrows in a and $\mathbf{c}$ indicate marginal narrow area, which is not stained with silver nitrate. Scales were stained with silver nitrate. G, groove. Bars: $50 \mu \mathrm{m}$

Fig. 4 Light microscopy images of frontal sections of 7-day regenerating scales. a A scale regenerated in the control condition, $\mathbf{b}$ a scale regenerated in the control condition and incubated for 4 hours in physiological saline, c a scale regenerated in the CaDPD condition, $\mathbf{d}$ a scale regenerated in the CaDPD condition and incubated for 4 hours in physiological saline. Arrows in c and d indicate pores and concavities. All sections were stained with silver nitrate and methylene blue-azure II. EL, External layer; BP, basal plate. Bars: $50 \mu \mathrm{m}$

Fig. 5 Transmission electron micrographs of the external layer of 7-day regenerating scales. Five layers $(A-E)$ were distinguished in scales regenerated under the control condition (a), whereas only two layers $(B, E)$ were distinguished in scales regenerated under the CaDPD condition (b). Stained with uranyl acetate and lead citrate. Bars: $1 \mu \mathrm{m}$. The higher magnification images of each layer to compare collagen fibril diameter are shown in c-g. c the A layer, $\mathbf{d}$ the B layer, $\mathbf{e}$ the C layer, $\mathbf{f}$ the D layer, $\mathbf{g}$ the E layer. Stained with uranyl acetate and lead citrate. Bars: $0.2 \mu \mathrm{m}$

Fig. 6 Transmission electron micrographs of the external layer of 7-day regenerating scales. (a) A scale 
regenerated in the control condition, (b) a scale regenerated in the CaDPD condition, (c) a scale regenerated in the CaDPD condition and incubated for 4 hours in physiological saline. Arrows indicate putative mineral deposits. Unstained. Bars: $0.5 \mu \mathrm{m}$ 
Table 1 Composition (g) of diets used

\begin{tabular}{lcc}
\hline Ingredients & Control & CaDPD \\
\hline Egg albumin $^{\mathrm{a}}$ & 40 & 40 \\
Dextrin $^{\mathrm{P}}$ & 15 & 15 \\
Potato starch $^{\mathrm{b}}$ & 15 & 15 \\
Soy bean oil $^{\mathrm{c}}$ & 3 & 3 \\
Cod liver oil $_{\text {Cellulose }}$ & 2 & 2 \\
Ca-lactate & 6.05 & 22.01 \\
$\mathrm{~K}_{2} \mathrm{HPO}_{4}$ & 9.0 & 0 \\
$\mathrm{Na}_{2} \mathrm{H}_{2} \mathrm{PO}_{4}$ & 4.75 & 0 \\
Vitamin mixture $^{\mathrm{d}}$ & 1.76 & 0 \\
$\mathrm{Mineral} \mathrm{mixture}^{\mathrm{e}}$ & 2 & 2 \\
\hline
\end{tabular}

${ }^{\text {a }}$ Commercial egg albumin denatured by boiling with ethanol and then defatted with ethyl ether

${ }^{\mathrm{b}}$ Boiled into $\alpha$-starch

${ }^{\mathrm{c}}$ Vitamin E (40 mg/100 g diet) added

${ }^{\mathrm{d}}$ After Halver and Coastes [30]

${ }^{\mathrm{e}}$ Mineral mixture consisting of $\mathrm{NaCl}$ (29.648\%), $\mathrm{MgSO}_{4} \cdot 7 \mathrm{H}_{2} \mathrm{O}$ (56.0\%), Fe-citrate (12.0\%), $\mathrm{AlCl}_{3} \cdot 6 \mathrm{H}_{2} \mathrm{O}$ (0.072\%), $\mathrm{ZnSO}_{4} \cdot 7 \mathrm{H}_{2} \mathrm{O}$ (1.428\%), $\mathrm{MnSO}_{4} \cdot 4-6 \mathrm{H}_{2} \mathrm{O}(0.032 \%), \mathrm{CuCl}(0.044 \%), \mathrm{KI}(0.068 \%), \mathrm{CoCl}_{2} \cdot 6 \mathrm{H}_{2} \mathrm{O}$ $(0.420 \%)$ 


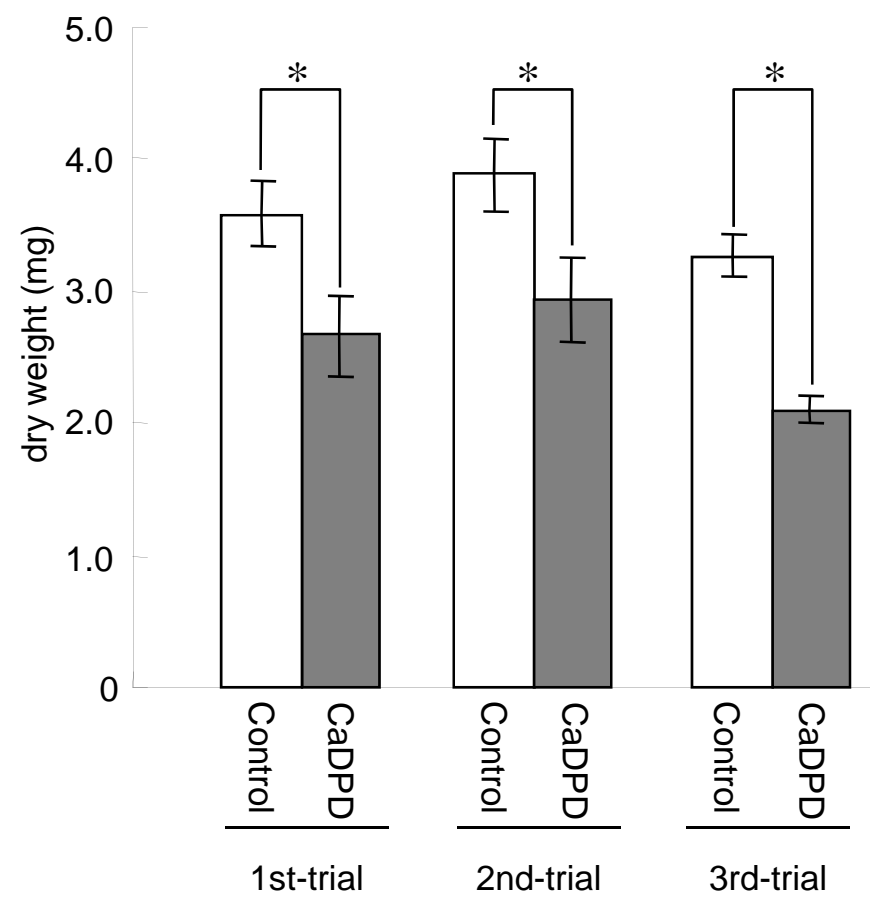

Fig. 1

Ogawa et al 
(a) 1st-trial

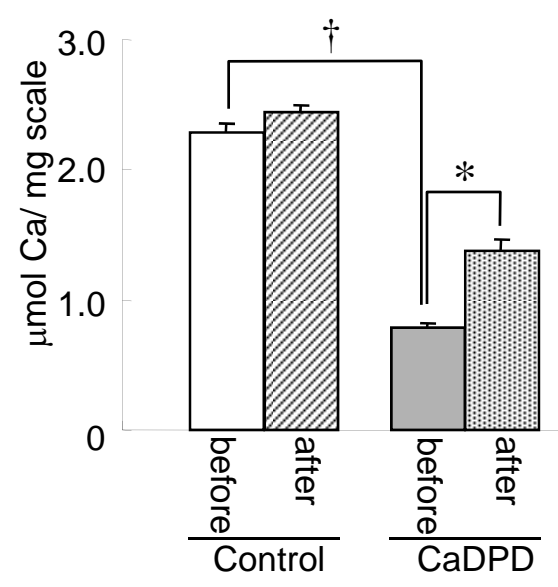

(b) 2nd-trial

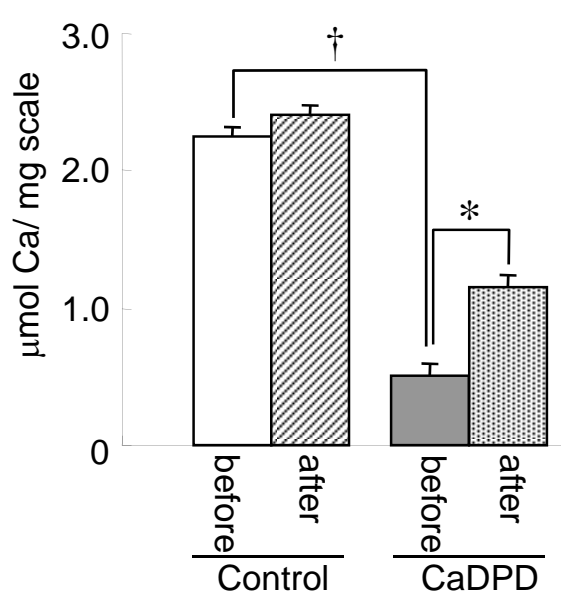

(c) 3rd-trial

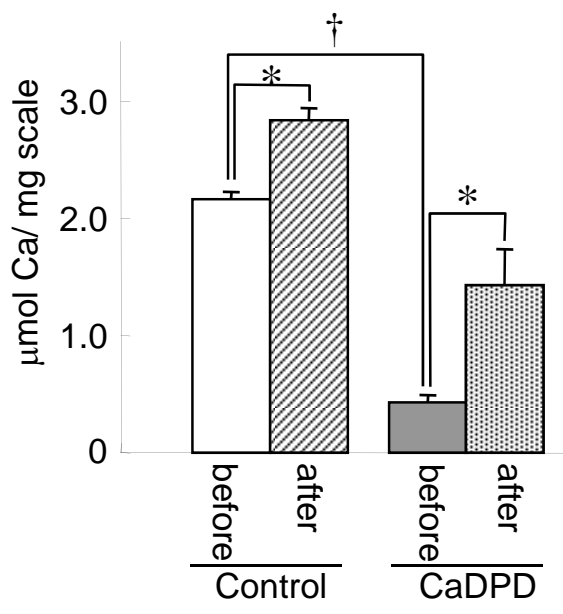

(d) 1st-trial

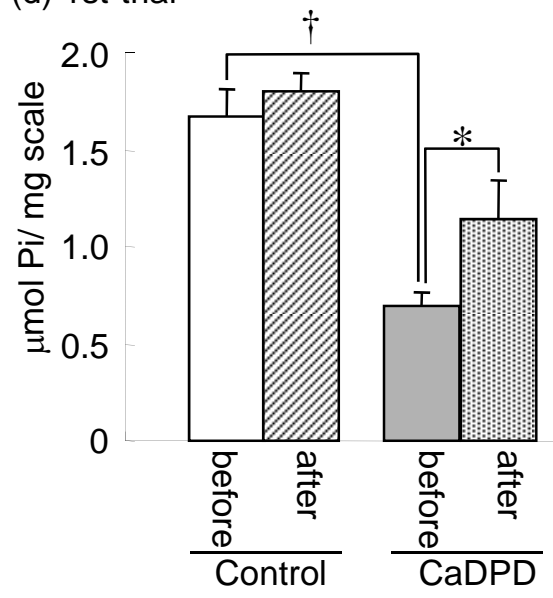

(e) 2nd-trial

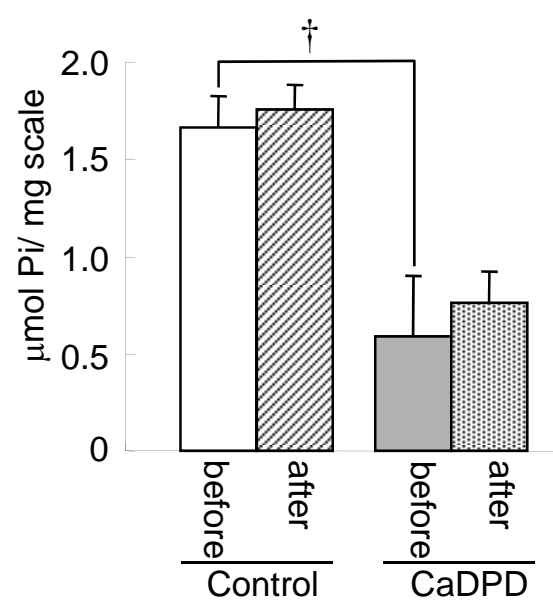

(f) 3rd-trial

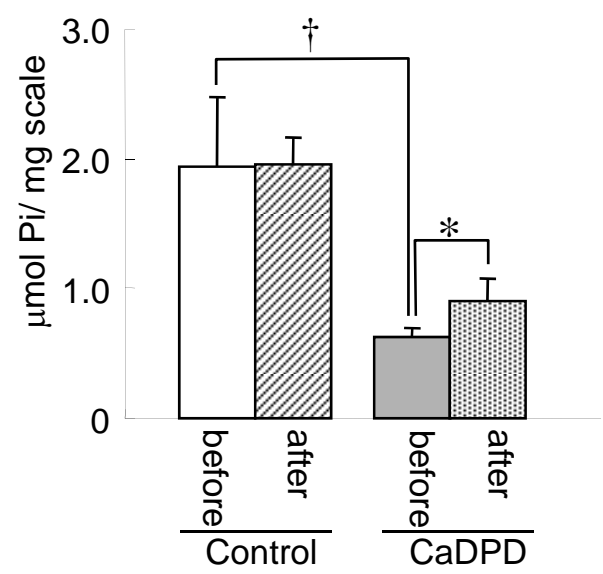

Fig. 2

Ogawa et al 
(a)

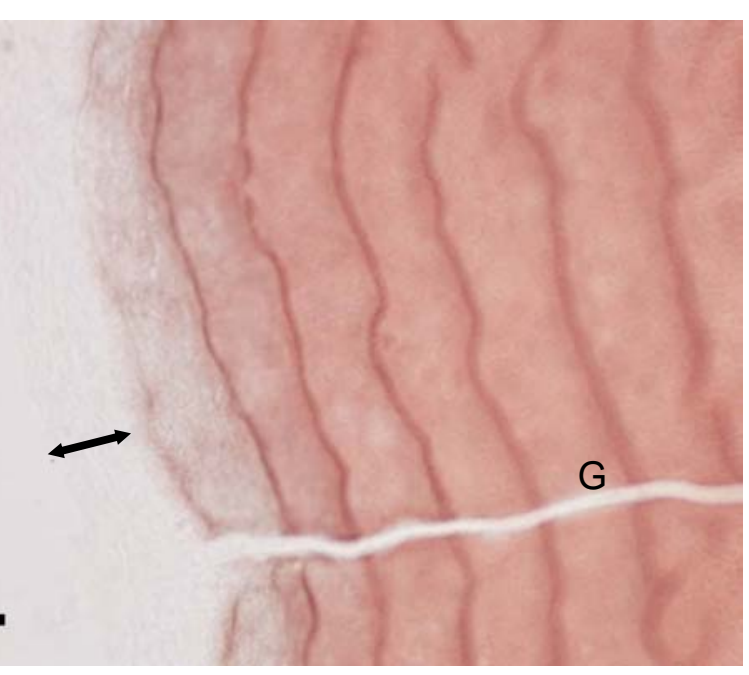

(c)

G (b)

G

(d)

\section{G}

G

Fig. 3

Ogawa et al 
(a)

(b)

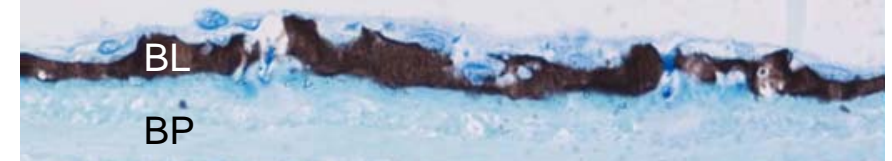

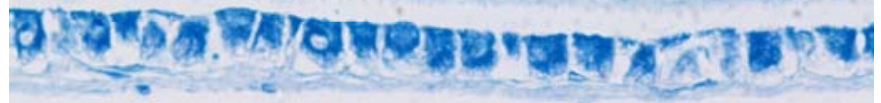

(c)

(d)

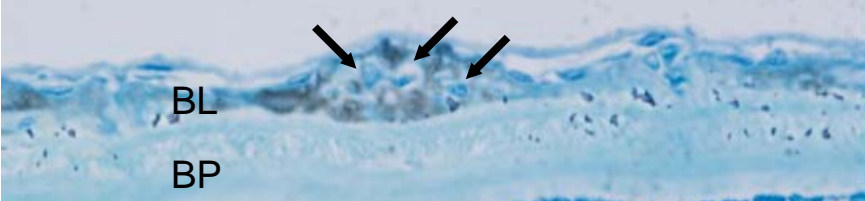

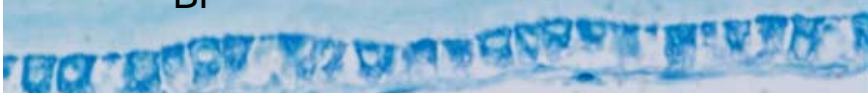

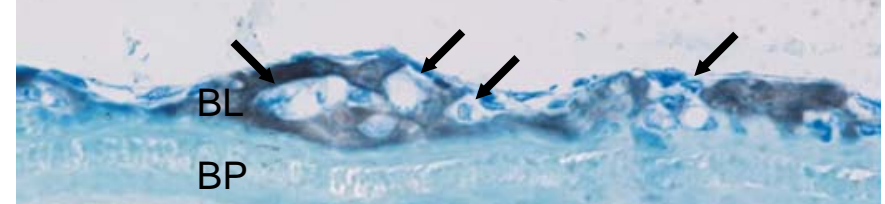

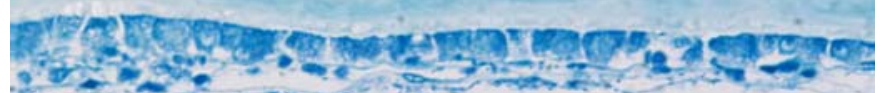
$\rightarrow-2 y$.

Fig. 4

Ogawa et al 


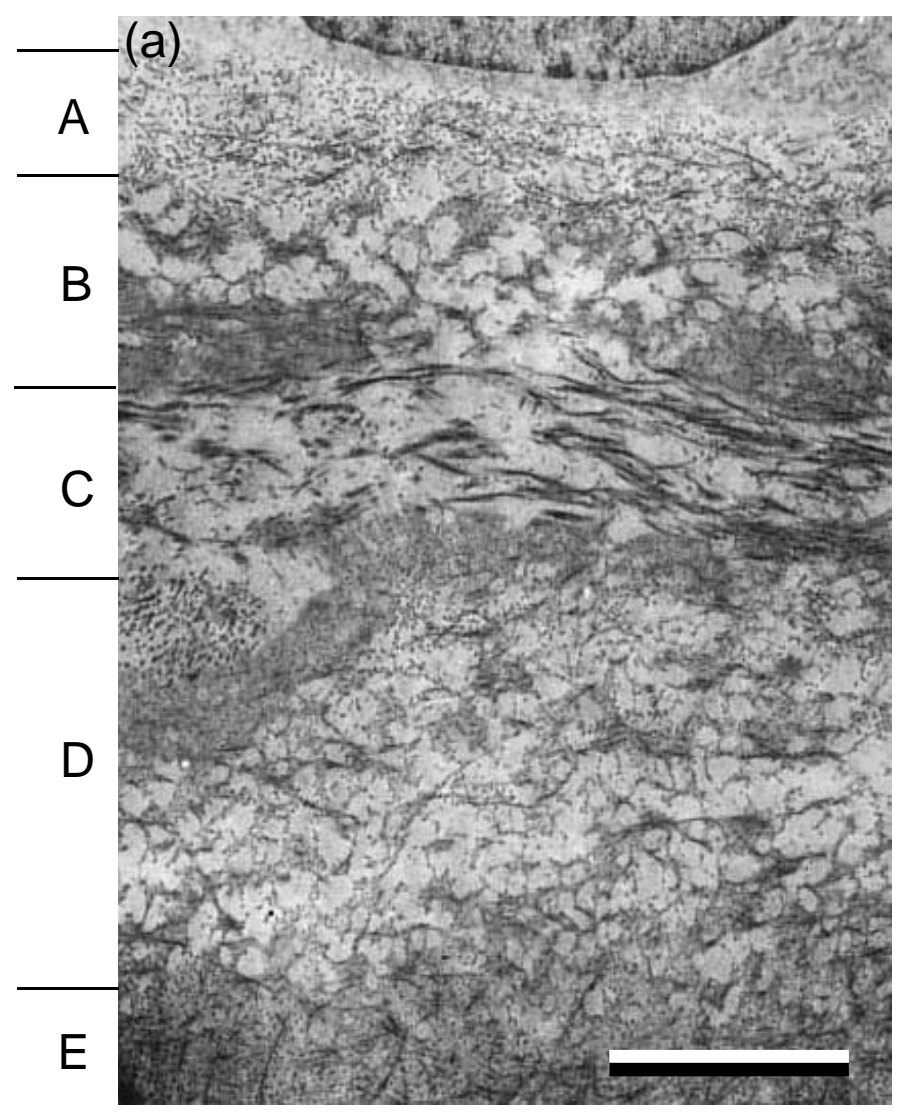

(b)

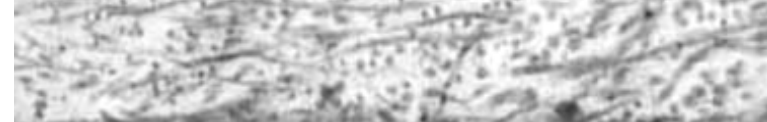

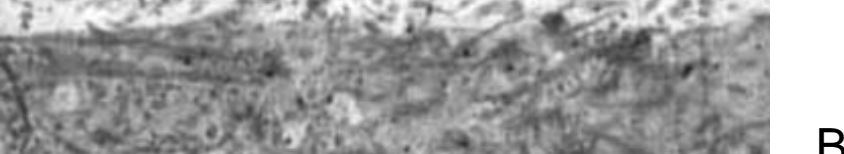

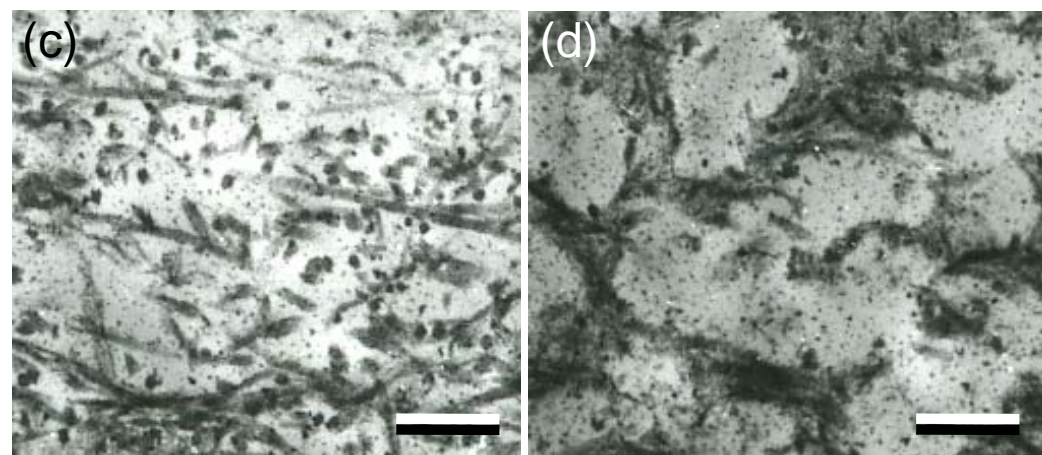

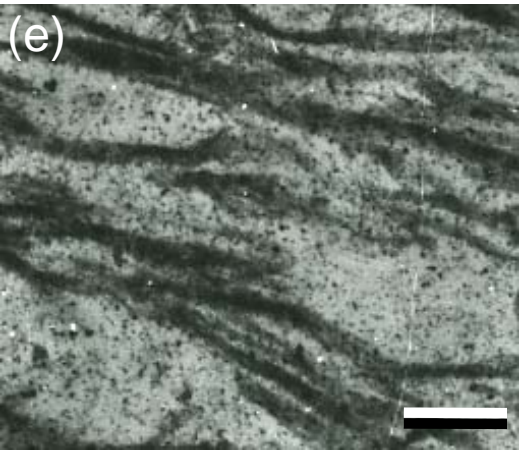

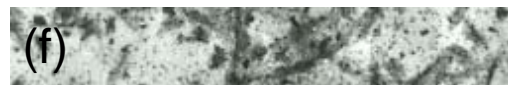
fivis.

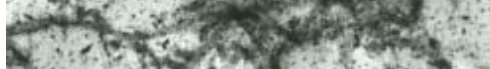

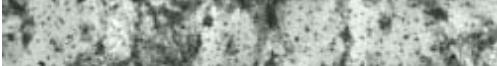

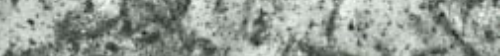

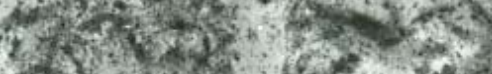
35 .

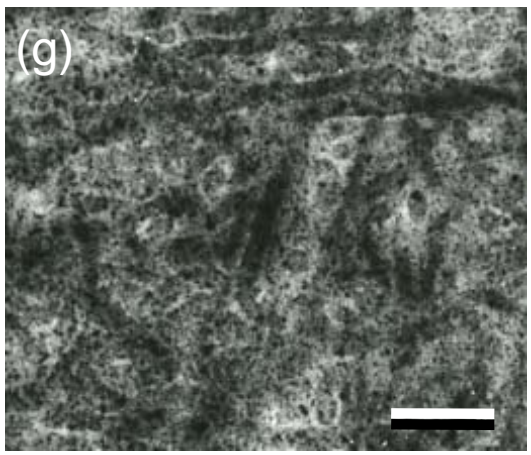

Fig. 5

Ogawa et al 


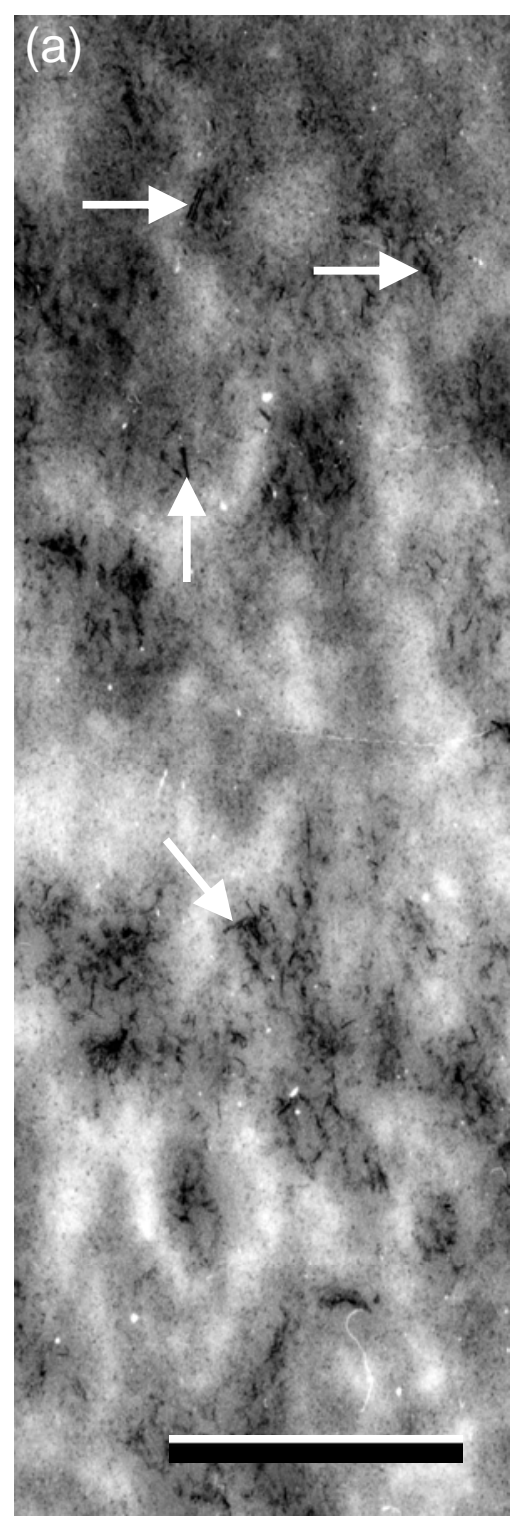

(b)

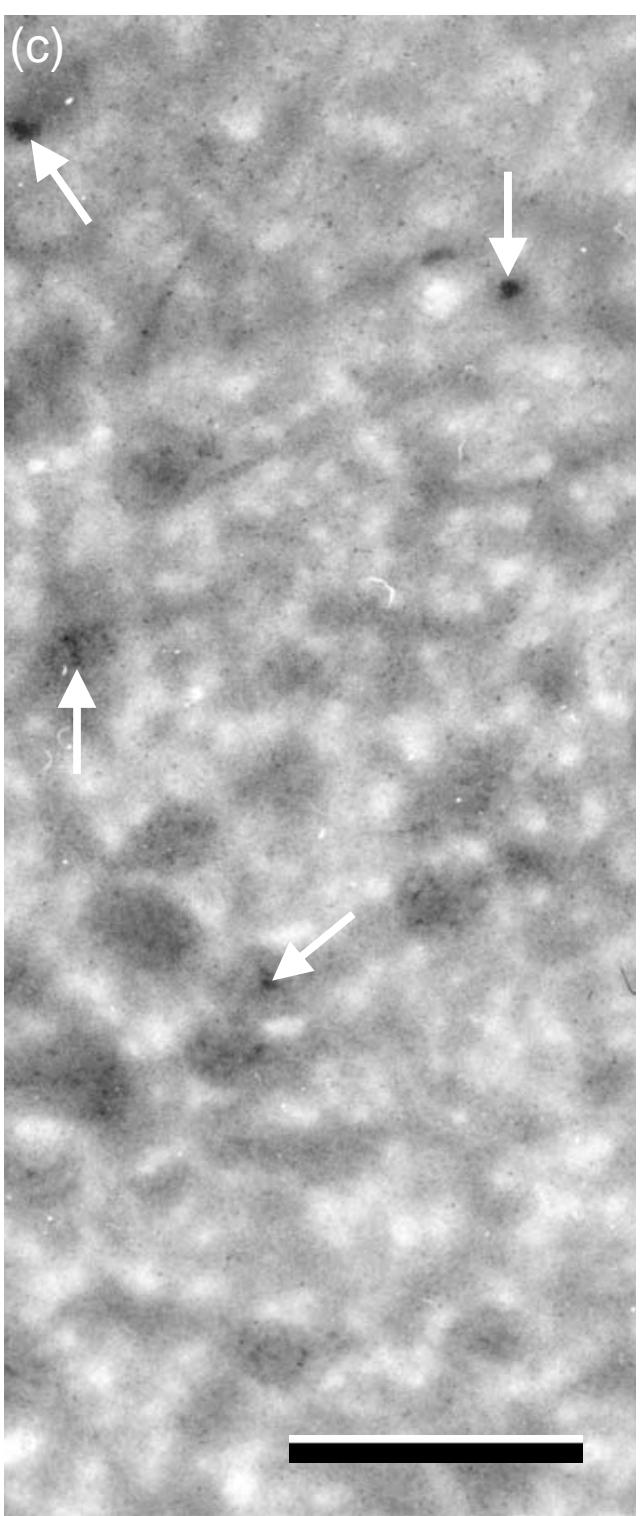

Fig. 6

Ogawa et al 